\title{
The anesthetic management of a patient with Emery-Dreifuss muscular dystrophy for orthopedic surgery
}

\author{
[La prise en charge anesthésique d'un patient de chirurgie orthopédique atteint de \\ dystrophie musculaire de Emery-Dreifuss]
}

Robin James Aldwinckle BMBS, Alison Sylvia Carr MBBS FRCA

Purpose: To report a patient with Emery-Dreifuss muscular dystrophy (EDMD) coming for an orthopedic procedure, with potential problems of sudden cardiac death, difficult airway, and neuromuscular disorders who was managed successfully by permanent pacemaker insertion, total iv anesthesia (TIVA), laryngeal mask insertion (LMA) insertion and continuous epidural blockade.

Clinical features: A 22-yr-old man with known EDMD presented for triple arthrodesis of his right foot and fractional lengthening of his hamstrings bilaterally. Anesthesia was induced with a TIVA technique, and maintained throughout the operative period. A suspected difficult airway was managed by the use of a LMA, and analgesia for the peri-, and postoperative period provided by a continuous epidural infusion. The patient's perioperative course was uneventful.

Conclusion: EDMD is a rare disorder. However, anesthesia is often required for orthopedic procedures. This case report illustrates the many potential difficulties that may be encountered. Regional anesthesia combined with light general anesthesia offers a method of avoiding many of these difficulties.

Objectif : Présenter le cas d'un patient, atteint de dystrophie musculaire de Emery-Dreifuss (DMED), qui devait subir une intervention orthopédique. Cette opération comportait des risques potentiels de mort cardiaque soudaine, d'intubation difficile et de troubles neuromusculaires dont la prise en charge a été réussie grâce à l'insertion d'un stimulateur cardiaque permanent, à l'anesthésie exclusivement iv (AENV), à l'utilisation d'un masque laryngé (ML) et d'un blocage péridural continu.

Éléments cliniques : Un homme de 22 ans, dont la DMED était connue, devait subir une triple arthrodèse du pied droit et l'allongement fractionné bilatéral des ischio-jambiers. L'anesthésie a été induite selon une technique AEIV qui a été maintenue tout au long de l'opération. L'usage d'un ML, et d'une perfusion péridurale continue pour l'analgésie périopératoire et postopératoire, ont permis de contourner les difficultés d'intubation soupçonnées. Aucun incident périopératoire n'est survenu.

Conclusion : La DMED est une affection rare. La présente observation illustre les nombreuses difficultés anesthésiques possibles dans les circonstances. L'anesthésie régionale, combinée à une anesthésie générale légère, représente une méthode efficace de contourner ces difficultés.

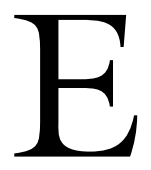

MERY-DREIFUSS muscular dystrophy (EDMD) is rare, although the third most common of the X-linked recessive muscular dystrophies. Although it was first described in the early 1900s, EDMD was not clearly delineated as a separate disease until the $1960 \mathrm{~s}^{1,2}$ Although the true frequency of the disorder is unknown it has been estimated to have an incidence of approximately $1: 100,000$ in the US. There have only been two case reports in the English anesthetic literature. ${ }^{3,4}$ Both cases reported the use of spinal anesthesia, although Jensen ${ }^{3}$ noted a history of previous general anesthesia for an appendicectomy.

EDMD typically presents at the age of four to five years with flexion contractures, and is characterized by the development of contractures of the elbows, Achilles tendon (causing toe walking) and posterior cervical muscles; muscle weakness in a humeroperoneal distribution; and cardiomyopathy usually presenting as heart block.

There are several important anesthetic considerations with these patients:

From the Department of Anaesthesia, Derriford Hospital, Derriford Hospital, Plymouth, UK.

Address correspondence to: Dr. Alison Sylvia Carr, Department of Anaesthesia, Derriford Hospital, Plymouth, UK. Phone: 01752

763393; Fax: 01752 763287; E-mail: alison.carr@phnt.swest.nhs.uk

Accepted for publication November 8, 2001.

Revision accepted January 18, 2002. 


\section{(i) Cardiac involvement}

Atrial conduction defects with a bradycardia are considered the hallmark of EDMD cardiomyopathy. ${ }^{5}$ Asymptomatic patients often show low amplitude $P$ waves and first degree heartblock. These changes may progress to atrial fibrillation. Conduction disturbances may also involve the infranodal conduction system resulting in slow junctional escape rhythms or complete heart block. ${ }^{6,7}$ Atrial standstill is a pathognomonic finding, ${ }^{8}$ with a lack of atrial response to intracardiac electrical or mechanical stimulation. ${ }^{9}$ Cardiomyopathy with poor left ventricular function occurs less commonly. The degree of cardiac involvement does not correlate with the degree of skeletal muscle involvement. Temporary (or permanent) cardiac pacing must be available at all times perioperatively, and myocardial depressant agents should be avoided in patients with significant cardiomyopathy.

\section{(ii) Neck stiffness}

Neck flexion may be markedly reduced in patients with EDMD, and hypoplasia of the third to the fifth vertebral bodies and intervertebral discs with hypophyseal joint fusion has been described. ${ }^{10}$ Endotracheal intubation may be difficult.

\section{(iii) Flexion contractures}

In addition to cervical flexion contractures there may also be involvement of the lumbar paravertebral muscles, making spinal/epidural anesthesia difficult. ${ }^{3}$ Care must also be taken when positioning the patient because of the multiple joint contractures.

\section{(iv) Muscle involvement}

Suxamethonium is contraindicated because of the risk of an exaggerated hyperkalemic response. Although malignant hyperthermia is considered a risk, it has not yet been reported in association with the disease.

We present a patient with EDMD undergoing triple arthrodesis of the right foot, using a laryngeal mask airway (LMA) and total iv anesthesia (TIVA) combined with epidural anesthesia.

\section{Case report}

A 22-yr-old man with known EDMD presented for triple arthrodesis of his right foot and fractional lengthening of his hamstrings bilaterally. There was a strong family history of EDMD with two of his cousins being affected. He was diagnosed with EDMD at five years with an unstable gait and repeated falls. On examination he had contractures of his elbows, Achilles tendons, and posterior cervical muscles. The two cousins had pacemakers inserted in their late teens because of conduction abnormalities. He was investigated by a cardiologist, at the age of 20 , following a brief history of dyspnea, and an electrocardiogram (ECG) at the time had showed atrial flutter with variable block. An echocardiogram had shown good left ventricular function (ejection fraction 64\%), with normal dimensions, and no evidence of cardiomyopathy. His cardiac rhythm was slow atrial flutter, and since EDMD may be complicated by atrial dysrrhythmias progressing to atrial standstill, a ventricular pacemaker (VVI mode) was inserted without complications. Warfarin was also prescribed because of the risk of thrombo-embolism secondary to atrial arrhythmias.

On physical examination he showed marked muscular wasting and weighed only $47 \mathrm{~kg}$. He had marked contractures of his elbows, Achilles tendons, and of the posterior cervical muscles. He also had marked wasting of biceps, triceps, quadriceps and calf muscles, yet in spite of this he was still able to walk. He also showed a marked fixed lumbar lordosis. His airway was assessed as Mallampati class I, but there was marked restriction of head movement with his neck almost fixed in extension. Blood pressure was $110 / 65$, and pulse was regular at $60 \mathrm{~min}$. Examination of the chest, and auscultation of the heart sounds were normal.

Hemoglobin, white cell count, platelet count, plasma electrolytes, prothrombin time, and activated partial thromboplastin time were normal. An ECG showed sinus rhythm of 60 beats. $\mathrm{min}^{-1}$, with first degree heart block, and a normal axis. A chest $x$-ray showed a normal heart size, and clear lung fields. After discussion with the cardiologist no further echocardiography was required, in view of his normal heart size on chest $x$-ray and previously normal ventricular function. Because of the presence of sinus rhythm on the ECG, and hence the low risk of thromboembolism, oral warfarin was stopped preoperatively to allow a regional anesthetic technique and reduce any perioperative bleeding.

The patient was very anxious, and requested a general anesthetic. The anesthetic plan was an epidural or regional block combined with a light TIVA. After discussion with the patient it was felt that it would be preferable to insert an epidural after induction.

In view of the marked lumbar lordosis, and the inflexibility of the spine, we were not confident that an epidural technique would be possible. In view of this possibility general anesthesia combined with regional anesthesia was chosen over regional anesthesia alone. Oral premedication consisted of temazepam $(30 \mathrm{mg})$ and metoclopramide $(10 \mathrm{mg})$. In the anesthetic room the patient was drowsy, but cooperative. Monitors applied before induc- 


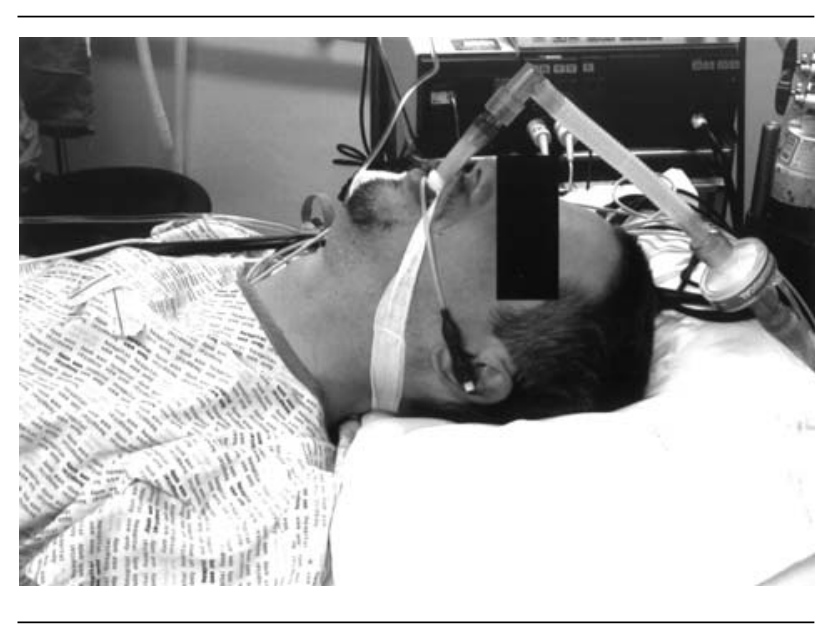

FIGURE The laryngeal mask in place. Note the fixed neck extension, and decreased thyromental distance.

tion were an automated blood pressure cuff, an ECG, and a pulse oximeter. Anesthesia was induced with fentanyl $(50 \mu \mathrm{g}) i v$ and a target controlled infusion (TCI) of propofol. The TCI infusion was initially set at $4 \mu \mathrm{g} \cdot \mathrm{mL}^{-1}$, but was increased to $6 \mu \mathrm{g} \cdot \mathrm{mL}^{-1}$ to allow a smooth induction. Manual ventilation of the lungs was easy, and, prior to LMA insertion, laryngoscopy was performed. Only the epiglottis could be seen (grade 3 laryngoscopy). A size 4 laryngeal mask was inserted easily at the first attempt and provided an excellent airway through which the patient breathed spontaneously an oxygen/air mixture (Figure). Lumbar epidural was performed in the left lateral position at the L3-4 interspace. The spine was rigid, and, in the best position for insertion of a lumbar epidural, had a marked lumbar lordosis. Nevertheless the epidural was inserted easily at the first attempt. An epidural test dose of $3 \mathrm{~mL}$ of plain $0.5 \%$ bupivacaine was injected, and a further $7 \mathrm{~mL}$ of plain $0.5 \%$ bupivacaine injected preoperatively.

During surgery the patient was in the supine position. The intraoperative course was uneventful with stable blood pressure, pulse, oxygen saturation, and temperature. Anesthesia was maintained with a propofol TCI at a concentration of $3 \mu \mathrm{g} \cdot \mathrm{mL}^{-1}$, and an epidural infusion of $0.167 \%$ bupivacaine $\left(8 \mathrm{~mL} \cdot \mathrm{hr}^{-1}\right)$ maintained analgesia. The procedure lasted three hours.

\section{Discussion}

This case report describes general anesthesia in a man with EDMD and highlights a number of potential anesthetic problems.

The cardiac conduction abnormalities associated with EDMD, typically atrial conduction defects and bradycardia, had been managed by the insertion of a VVI pacemaker two years previously. Our patient had no evidence of cardiomyopathy or heart failure.

Airway management was potentially difficult. There was almost no neck movement and a decreased thyromental distance $(3 \mathrm{~cm})$, but a normal Mallampati (I) score. Fortunately the fixed position of his neck was in an exaggerated "sniffing the morning air" position, and this allowed the LMA to be inserted easily and to provide an excellent airway. Although intending to use the LMA for airway management, we performed laryngoscopy after induction of anesthesia to determine how difficult tracheal intubation would be should it be required perioperatively, or should an LMA be contraindicated in future cases. At laryngoscopy (albeit without muscle relaxation), there was only visualization of the epiglottis. Intubation in these circumstances would have required a bougie, intubating LMA or other technique. If a patient is considered at high risk for regurgitation and inhalational pneumonia, awake intubation with the use of a fibreoptic laryngoscope is recommended.

Airway management with the LMA in patients with EDMD may be questioned on the basis of potential abnormalities with gastric emptying. It is unknown whether there is delayed gastric emptying in patients with EDMD, although in patients with Duchenne muscular dystrophy (DMD) delayed gastric emptying has been reported. ${ }^{11}$ In view of the potential airway problems in EDMD, the method of airway management will be a question of judgement in each case, taking into account any risk of regurgitation from the history, and the difficulty in tracheal intubation. Pharmacological prophylaxis with a pro-kinetic agent would seem prudent with the premedication in any case, regardless of the ultimate method of airway management.

The epidural catheter was inserted in the patient under general anesthesia. Although regional anesthesia is usually best performed in the awake patient to minimize the risks of neural injury, patient refusal is an absolute contraindication. Insertion under general anesthesia also allowed optimum positioning of the patient, which may be uncomfortable in the awake patient with joint contractures.

Preoperative assessment in this case indicated that regional anesthesia would be virtually impossible, due to the patient's marked lumbar lordosis, and rigid spine due to flexion contractures. However, because of the huge potential benefits of an epidural it was attempted, and was easily inserted. A regional technique provided excellent perioperative analgesia, avoiding opioid use and its associated respiratory depressant effects. An epidural catheter technique was 
chosen over nerve blockade for a number of reasons. Firstly it allowed the provision of excellent peri, and postoperative analgesia. Secondly, because of the bilateral nature of the surgery, although nerve blocks could have been used, it would have required at least three blocks (bilateral sciatic blocks, and a saphenous block on the side of the arthrodesis) to ensure the same degree of analgesia. Thirdly postoperative analgesia would not be able to be as prolonged, and fourthly because of the frail nature of our patient potentially toxic doses of bupivacaine may have been reached with the nerve blocks. In long procedures it may be uncomfortable for the patient to remain still, especially if there are problems with joint contractures and positioning. A light general anesthetic in combination with a regional block may be the most appropriate option in such situations.

TIVA was chosen over an inhalational technique for a variety of reasons. Firstly, volatile agents are best avoided due to the possibility of triggering malignant hyperpyrexia $(\mathrm{MH})$ in a patient with a myopathy. ${ }^{12}$ To date $\mathrm{MH}$ has not been described in EDMD. Possible reasons are the rarity of the condition, combined with a paucity of publications about anesthesia in patients with EDMD or a low, and as yet unproven risk of $\mathrm{MH}$ (or an MH type reaction) in these patients. Secondly, it allowed rapid onset and offset of anesthesia, allowing any problems on recovery of consciousness to be assessed quickly, and also to decrease the incidence of postoperative nausea and vomiting. Suxamethonium is contraindicated in EDMD since it may cause an exaggerated hyperkalemic response. ${ }^{13}$ The non-depolarizing muscle relaxants are best avoided because of the possible delays in muscle function recovery (it is known that with the use of vecuronium in DMD there can be up to sixfold delay in the recovery of muscle function). ${ }^{14}$ The use of the LMA did allow us to avoid the use of muscle relaxants, which may complicate the management of patients with EDMD.

\section{Conclusion}

A patient suffering from EDMD presents a number of challenges to the anesthesiologist, most notably cardiac arrhythmias, cardiomyopathy, airway difficulties, and difficult access for regional anesthesia techniques due to flexion contractures. An epidural combined with TIVA provided excellent peri/postoperative analgesia and allowed the patient to recover rapidly from anesthesia.

\section{References}

1 Emery $A E H$, Dreifuss FE. Unusual type of benign Xlinked muscular dystrophy. J Neurol Neurosurg Psychiatry 1966; 29: 338-42.
2 Dreifuss FE, Hogan GR. Survival in X-chromosomal muscular dystrophy. Neurology 1961; 11: 734-7.

3 Jensen $V$. The anaesthetic management of a patient with Emery-Dreifuss muscular dystrophy. Can J Anaesth 1996; 43: 968-71.

4 Morrison P, Jago RH. Emery-Dreifuss muscular dystrophy. Anaesthesia 1991; 46: 33-5.

5 Voit T, Krogmann O, Lenard HG. Emery-Dreifuss muscular dystrophy: disease spectrum and differential diagnosis. Neuropediatrics 1988; 19: 62-71.

6 Oswald AH, Goldblatt J, Horak AR, Beighton P. Lethal cardiac conduction defects in Emery-Dreifuss muscular dystrophy. S Afr Med J 1987; 72: 567-70.

7 Yoshioka M, Saida K, Itagaki $\Upsilon$, Kamiya T. Follow up study of cardiac involvement in Emery-Dreifuss muscular dystrophy. Arch Dis Child 1989; 64: 713.

8 Fishbein MC, Siegel RJ, Thompson CE, Hopkins LC. Sudden death of a carrier of X-linked Emery-Dreifuss muscular dystrophy. Ann Intern Med 1993; 119: 900-5.

9 Disertori $M$, Guarnerio $M$, Vergara $G$, et al. Familial endemic persistent atrial standstill in a small mountain community: review of eight cases. Eur Heart J 1983; 4: 354-61.

10 Miller RG, Layzer RB, Mellenthin MA, Golabi M, Francoz RA, Mall JC. Emery-Dreifuss muscular dystrophy with autosomal dominant transmission. Neurology 1985; 35: 1230-3.

11 Smith CL, Bush GH. Anaesthesia and progressive muscular dystrophy. Br J Anaesth 1985; 57: 1113-8.

12 Brownell $A K W$. Malignant hyperthermia: relationship to other diseases. Br J Anaesth 1988; 60: 303-8.

13 Boltshauser E, Steinmann B, Meyer A, Jerusalem F. Anaesthesia-induced rhabdomyolysis in Duchenne muscular dystrophy (Letter). Br J Anaesth 1980; 52: 559.

14 Ririe DG, Shapiro F, Sethna NF. The response of patients with Duchenne's muscular dystrophy to neuromuscular blockade with vecuronium.

Anaesthesiology 1998; 88: 351-4. 